Check for updates

Cite this: RSC Adv., 2019, 9, 11621

Received 11th February 2019

Accepted 5th April 2019

DOI: 10.1039/c9ra01082c

rsc.li/rsc-advances

\section{Mesoporous carbon nanospheres deposited onto $D$-shaped fibers for femtosecond pulse generation $\uparrow$}

\author{
Fang Wang, ${ }^{a}$ Hua Zhou, ${ }^{a}$ Nan Li, ${ }^{a}$ Jiaxing Liu, ${ }^{a}$ Daguang Li, ${ }^{a}$ Zhe Kang, ${ }^{\text {ab }}$ Zhixu Jia, ${ }^{a}$ \\ Zhen-an Qiao, (D) ${ }^{a}$ Weiping Qin (D) ${ }^{a}$ and Guanshi Qin (ID *a
}

We demonstrate nonlinear optical modulation by combining mesoporous carbon nanospheres (MCNs) and D-shaped fibers (DFs). The MCNs are prepared by the silica-assisted strategy and the DFs are fabricated through a precision wheel polishing method. When the MCNs are deposited onto the DF as the saturable absorbers (SAs), the SAs possess broadband linear absorption and nonlinear saturable absorption properties. As the DF-MCNs SA is integrated into the laser cavities, ultrafast lasers at 1.56 and $2 \mu \mathrm{m}$ were realized with minimal pulse duration down to a few hundreds of femtoseconds. Compared with the film and microfiber-based MCNs-SAs, the DF-MCNs SAs exhibit greater robustness and stronger evanescent field, and are more effective at generating femtosecond pulses. Our results verify that DFMCNs as a kind of cost-effective and easily-prepared SA would be of great importance for stable and high-power femtosecond fiber lasers.

\section{Introduction}

Femtosecond fiber lasers exhibit many desirable advantages of extremely short temporal resolution, ${ }^{1}$ and high peak intensity, ${ }^{2}$ which make them receive much attention in the research fields of fundamental research, ${ }^{3,4}$ optical communication, ${ }^{5}$ medicine, ${ }^{6,7}$ and remote gas sensing. ${ }^{8,9}$ For the passive mode-locking technique, a nonlinear optical element, called a saturable absorber (SA), is mainly used to induce the generation of a femtosecond pulse. Over the past decades, carbon-based materials such as carbon nanotubes (CNTs) and graphene have been demonstrated for high-performance nonlinear SAs due to their outstanding physical and chemical properties. ${ }^{10-16}$ Compared with conventional laser mode-locking materials and devices, carbon-based materials have notable advantages such as (i) ultrashort recovery time, (ii) a wide operating bandwidth, (iii) a high thermal damage threshold, (iv) a simple preparation process, and (v) an extremely miniaturized footprint.

Mesoporous carbon nanospheres (MCNs), a new family of carbon-based materials, have shown many potential applications in a wide range of research fields due to the unique characteristics, such as good electrical conductivity, chemical inertness, high surface area, large porosity, and good

${ }^{a}$ State Key Laboratory on Integrated Optoelectronics, College of Electronic Science and Engineering, State Key Laboratory of Inorganic Synthesis and Preparation Chemistry, College of Chemistry, Jilin University, Changchun 130012, China. E-mail: qings@jlu. edu.cn

${ }^{b}$ Changchun Observatory, National Astronomical Observatories, CAS, Changchun 130117, China

$\dagger$ Electronic supplementary information (ESI) available. See DOI: 10.1039/c9ra01082c biocompatibility. ${ }^{17-23}$ Although MCNs have been widely studied and applied in many areas, few groups investigated and reported their nonlinear optical properties. Recently, our group demonstrated the nonlinear saturable absorption properties of MCNs and the application for the broadband pulsed lasers generation. ${ }^{24}$ Compared with usual SAs, MCNs show good dispersity and stability in aqueous solutions, which have advantages for fabricating the MCNs-SAs and stable mode-locked operation. ${ }^{24}$ However, in our previous work, the fabricated SA films were placed between two fiber connectors to form fiber-compatible SAs, and then integrated into the cavities. In spite of their simple fabrication, the optical absorption was weak due to the limited nonlinear interaction length. Furthermore, the absorption coefficients of MCNs were higher than those of graphene and CNTs, so the damage induced by photothermal effect or direct physical contact could potentially restrict the performance of the fiber laser, particularly at high power. We also used the MCNs deposited onto the microfiber as the SA with evanescent field, but the microfiber is not robust enough for the long-term operation, and the microfiber will be easy to introduce large loss and strong nonlinearity for the small core size. ${ }^{25-27}$ In order to overcome these drawbacks, an alternative method combining a D-shaped fiber (DF) and MCNs as the SAs has been proposed, which could improve the stability of femtosecond lasers for a longer nonlinear interaction length between the guided light and SAs and further enhance the damage threshold due to the weak evanescent wave interacting with SAs.

In this paper, we demonstrate an MCNs-SA based on DF fibers for the operation of femtosecond fiber lasers. The MCNs are synthesized by a "silica-assisted" method with an average size of $400 \mathrm{~nm}$ and then deposited onto the DF to form the SAs. The SAs 
show broadband linear and nonlinear absorption from visible to mid-infrared region. As the SAs are integrated into the $\mathrm{Er}^{3+}$-, and $\mathrm{Tm}^{3+}$-doped fiber laser cavities, mode-locked operations could be observed with the central wavelengths of 1559 and $1941.5 \mathrm{~nm}$, respectively. Our experimental results verify that deposition of MCNs onto DFs as SAs are promising broadband nonlinear candidates for the femtosecond pulses generation.

\section{Experimental section}

\subsection{Synthesis of the MCNs}

The MCNs used in our work were prepared by a facile and general "silica-assisted" strategy as described previously. ${ }^{24,28}$ The process uses phenolic resols as a polymer precursor, tetraethylorthosilicate (TEOS) as an inorganic precursor, and hexadecyl trimethylammonium chloride (CTAC) as a template. Firstly, a $10 \mathrm{~mL}$ amount of $25 \mathrm{wt} \%$ ammonia aqueous solution $\left(\mathrm{NH}_{4} \mathrm{OH}\right)$ was mixed with 4-8 $\mathrm{mL}$ of the solution containing absolute ethanol $(\mathrm{EtOH}), 1.04 \mathrm{~g}$ of the $25 \mathrm{wt} \%$ CTAC solution and $19 \mathrm{~mL}$ deionized water $\left(\mathrm{H}_{2} \mathrm{O}\right)$ in a breaker, then kept vigorous stirring for $30 \mathrm{~min}$. Subsequently, resorcinol ( $0.2 \mathrm{~g})$ was injected into reaction solution under continually stirring for $30 \mathrm{~min}$. Then $0.36 \mathrm{~mL}$ TEOS and $0.28 \mathrm{~mL}$ formaldehyde solution were introduced into the above-mentioned solution and stirred for $24 \mathrm{~h}$ at $30{ }^{\circ} \mathrm{C}$. The centrifugation was adopted to recover the solid product, which was then air-dried at $80{ }^{\circ} \mathrm{C}$ overnight. Calcination was carried out in a tubular furnace at $200,350,500,600{ }^{\circ} \mathrm{C}$ for $2 \mathrm{~h}$ and at $800^{\circ} \mathrm{C}$ for $5 \mathrm{~h}$ under $\mathrm{N}_{2}$ flow. The heating rate was $1{ }^{\circ} \mathrm{C} \min ^{-1}$ below $600{ }^{\circ} \mathrm{C}$ and $5{ }^{\circ} \mathrm{C} \min ^{-1}$ above $600{ }^{\circ} \mathrm{C}$. The carbon/silica nanocomposite was carbonized by etching of the silica, resulting in the formation of MCNs finally. The spherical diameters of MCNs were controlled by simply varying the ethanol concentration.

\subsection{Characterization of MCNs}

It was obvious that a large number of MCNs were synthesized successfully (Fig. S1†). Transmission electron microscopy (TEM) was used to characterize the synthesized MCNs at different scales (500 $\mathrm{nm}$ and $50 \mathrm{~nm}$ ), as shown in Fig. 1a and b. The monodispersed MCNs with an average particle size of $400 \mathrm{~nm}$ as the ethanol/water volume ratio was $1: 2.75$. The remarkable mesoporous structure was also observed. In order to further explore the properties of the MCNs, the linear absorption was measured by using the spectrophotometer (UV3600 Shimadzu). As depicted in the Fig. 1c, it was apparent that MCNs exhibited a stronger absorption than the solid carbon nanospheres (SCNs) ranging from 400 to $3400 \mathrm{~nm}$. Therefore, unique mesoporous structure and high surface area of MCNs are likely responsible to their high absorption. Moreover, the prepared MCNs using "silica-assisted" strategy can be dispersed in water by sonification to form stable colloidal suspensions, which have remained stable over 10 days without sign of agglomeration as shown in the inset of Fig. 1c and S2. $\dagger$ Fig. 1d showed the Raman spectrum of the MCNs. The $\mathrm{sp}^{3}$ and $\mathrm{sp}^{2}$ hybrid method of the MCNs were assigned by the two obvious peaks at 1340 and $1590 \mathrm{~cm}^{-1}$, which revealed that MCNs possessed an amorphous nature.

\subsection{Optical properties of DF-MCNs SA}

The DF used in our work was fabricated by getting rid of a part of the cladding from a single-mode fiber using a precision wheel polishing method. ${ }^{29}$ Meanwhile, the effective refractive index was reduced, resulting in mode broadening at the D-shaped region. When the nonlinear optical materials were deposited onto the DF, the propagating light can interact with them without being blocked in the fiber. So strong evanescent field between the light and nonlinear optical materials could be introduced to realize the femtosecond pulse generation. The schematic diagram of the MCNs-SA based on a DF was shown in Fig. 2a. Firstly, $1 \mathrm{mg}$ MCNs was mixed with $4 \mathrm{~mL}$ deionized water, and then kept sonification for $30 \mathrm{~min}$. A small amount of the solution was dropped onto the flat surface of the prepared DF. Finally, the consequent formation of the MCNs layer was deposited onto the polished region. The distance between the flat side and the fiber core was measured to be $\sim 6 \mu \mathrm{m}$, as shown in Fig. $2 \mathrm{~b}$. To verify the effect of the DF coated with MCNs, we measured its absorption spectrum using a supercontinuum light source (NKT Photonics Superk COMPACT) with a bare DF as the reference sample. Interestingly, the SA showed broadband linear absorption covering from 600 to $2400 \mathrm{~nm}$ in the Fig. 2c. The nonlinear saturable absorption properties of the SA were also characterized. The broadband absorption of the DFMCNs SA was not only caused by the strong absorption of the MCNs, but also effected by the DF. With the increasing of the wavelength, the effective refractive index was reduced and the mode area would be increased at the D-shaped region. Meanwhile, stronger evanescent field between the light and nonlinear optical materials could be introduced. So the absorbance of DF-MCNs SA at $1.5 \mu \mathrm{m}$ become much lower than that at $2.0 \mu \mathrm{m}$. We investigated the evolution of transmission ratio as the increase of the pump peak density, which then was fitted using the following equation: ${ }^{30}$

$$
\alpha(I)=\frac{\alpha_{\mathrm{S}}}{1+I / I_{\mathrm{S}}}+\alpha_{\mathrm{NS}}
$$

The measured data and well fitted line were shown in the Fig. 2d using a home-made pulsed fiber laser at $2000 \mathrm{~nm}$. The modulation depth $\alpha_{\mathrm{S}}$ and saturable intensity $I_{\mathrm{S}}$ were determined to be $1.92 \%$ and $15.1 \mathrm{MW} \mathrm{cm}^{-2}$, respectively. Using the same method, the saturable absorption properties were also measured at $1560 \mathrm{~nm}$ (modulation depth of 6.91\%, saturable power density of $13.6 \mathrm{MW} \mathrm{cm}^{-2}$ ) as shown in Fig. 2e. The abovementioned results showed that MCNs deposited onto DFs could be used as SAs for constructing broadband femtosecond fiber lasers at 1.56 and $2 \mu \mathrm{m}$.

\section{Results and discussion}

\subsection{Femtosecond pulses generation at $2 \mu \mathrm{m}$}

Femtosecond fiber lasers operating around $2 \mu \mathrm{m}$ have attracted huge interest due to the significant applications ranging from medicine and molecular spectroscopy, to molecular spectroscopy and mid-infrared sources. ${ }^{31-33}$ Therefore, we firstly integrated the DF-MCNs SA into a $\mathrm{Tm}^{3+}$-doped fiber laser (TDFL) cavity to verify whether the mode-locked laser operation or not, as shown in Fig. 3a. The laser cavity consisted of a $1570 \mathrm{~nm} \mathrm{Er}^{3+}$ - 
a

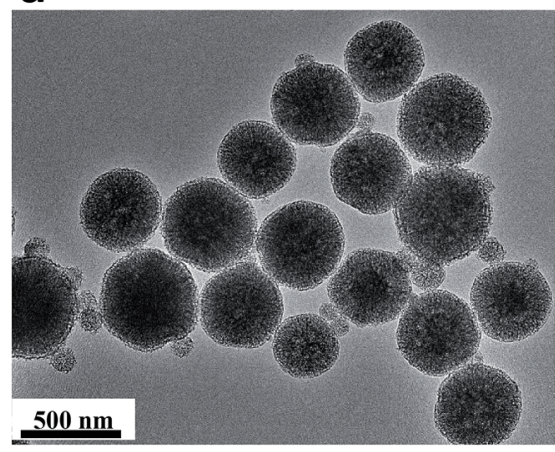

C

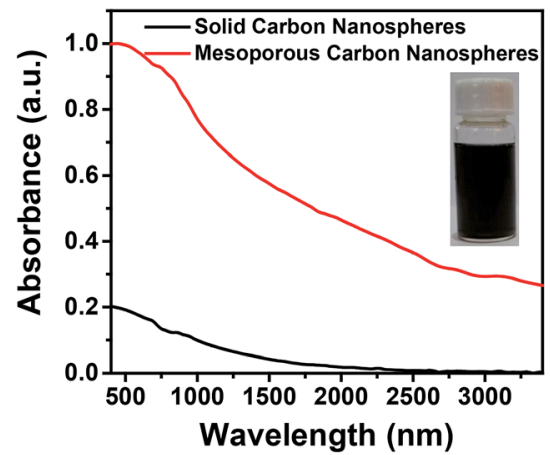

b

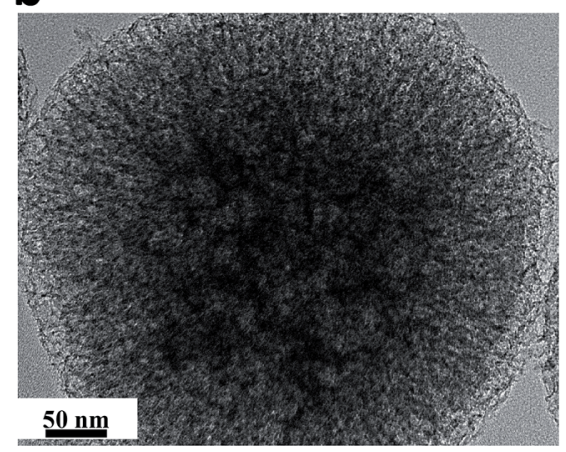

d

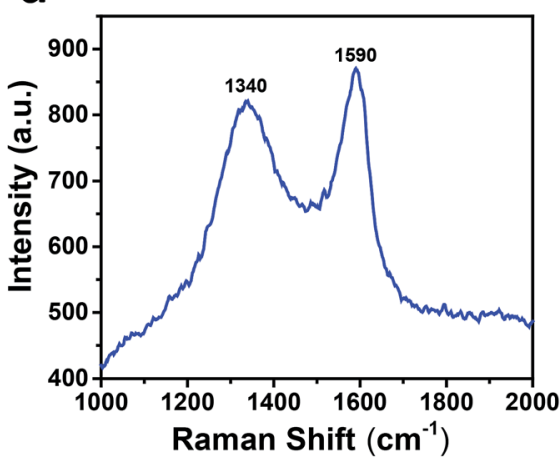

Fig. 1 ( $a$ and b) TEM images of the synthesized MCNs at different scales (500 nm and $50 \mathrm{~nm}$ ). (c) The absorption spectra of the MCNs and SCNs. Inset: the photograph of an aqueous solution of MCNs after 10 days. (d) The Raman spectrum of the MCNs.
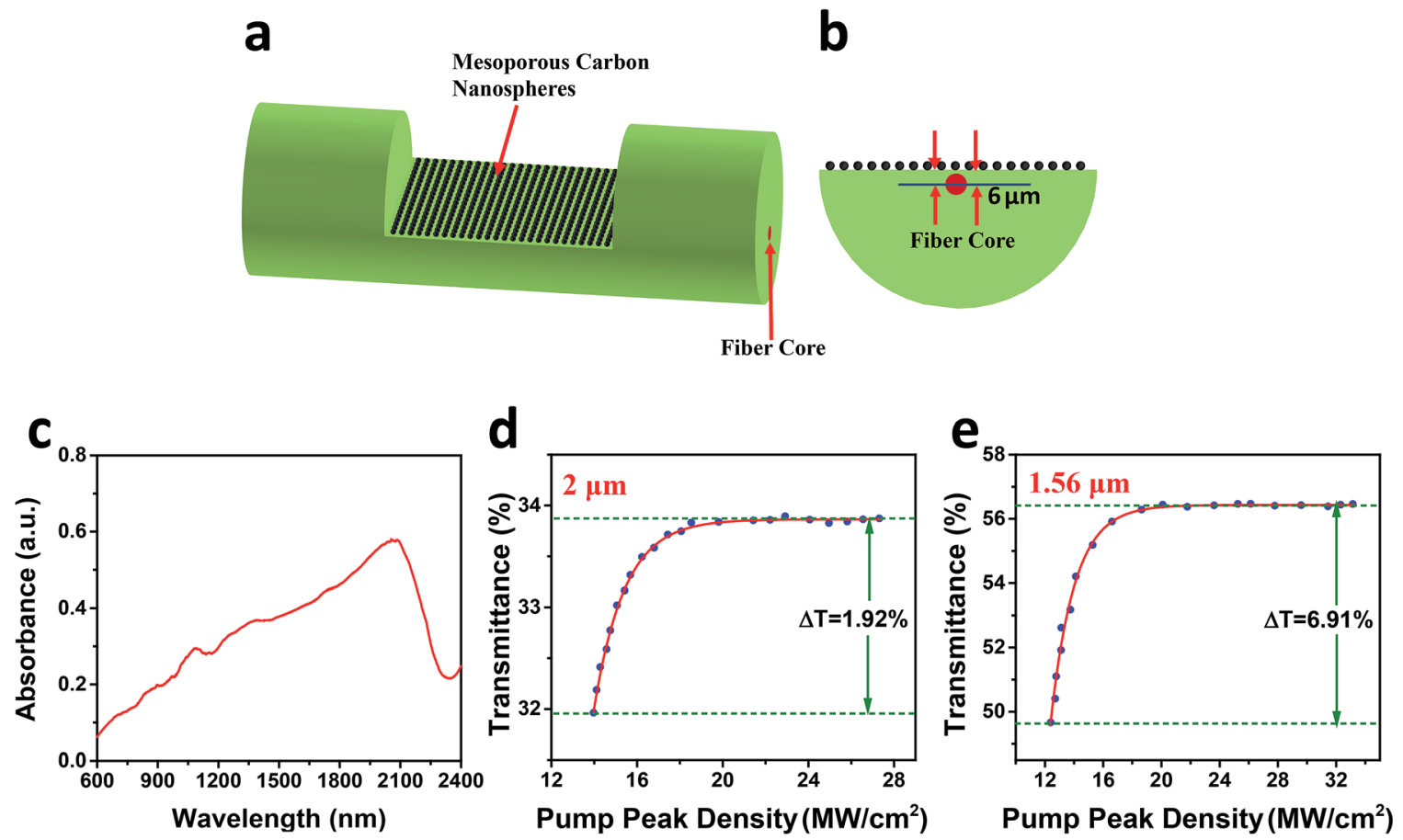

Fig. 2 (a) Schematic of the MCNs-SA based on a DF. (b) Cross-sectional structure of the DF. (c) The absorption spectrum of the DF-MCNs SA. (d and e) The optical nonlinear transmission data of the DF-MCNs SA at $2000 \mathrm{~nm}$ and $1560 \mathrm{~nm}$, respectively. 
doped fiber laser (EDFL) as the pump source. A 1550/2000 nm wavelength-division multiplexing (WDM) coupler was used to launch the pump light into the cavity. The gain medium was the single mode Tm-doped silica fiber (Nufern, SM-TSF-5/125) with the length of $20 \mathrm{~cm}$ and the dispersion parameter of $-12 \mathrm{ps}^{2}$ $\mathrm{km}^{-1}$ at $1.9 \mu \mathrm{m}$. To avoid any harmful feedbacks and guarantee the unidirectional laser propagation, an isolator (ISO) was added in the cavity. The intra-cavity polarization was adjusted by a polarization controller (PC). The $10 \mathrm{~dB}$ WDM coupler was adopted to output $10 \%$ of the laser and the rest continued propagating in the cavity. All fiber pigtails of the components we used in the experiment were made of single mode fibers (Coring SMF-28e) with a dispersion of $-67 \mathrm{ps}^{2} \mathrm{~km}^{-1}$ at $1.9 \mu \mathrm{m}$. The total length of the ring cavity was $\sim 7.8 \mathrm{~m}$, corresponding to a repetition rate of $25.5 \mathrm{MHz}$. The total dispersion in the cavity was calculated to be $-0.47 \mathrm{ps}^{2}$. The output lasers were analyzed by using an optical spectrum analyzer (YOKOGAWA, AQ6375), a digital oscilloscope (Tektronix, DPO70604C) and an autocorrelator (Alinar-lab, HAC-200).
If the MCNs were not deposited onto the DF, the laser was insensitive to the polarization state and always worked in the continuous wave regime with increase of the pump power. As the DF-MCNs SA was inserted into the cavity, mode-locked operation was observed with increase of the pump power to $1.3 \mathrm{~W}$ due to the evanescent field interaction. Fig. $3 \mathrm{~b}$ shows the measured spectrum of the mode-locked TDFL at a pump power of $2.1 \mathrm{~W}$. The central wavelength was located at $\sim 1941.5 \mathrm{~nm}$ and the spectral width at half-maximum was about $5.71 \mathrm{~nm}$. Obviously, Kelly bands induced by the interference of dispersion waves were also observed, which were the typical feature of the solitary laser operation. As shown in the Fig. 3c, the fiber laser delivered the pulse train with the time interval $\sim 39.2 \mathrm{~ns}$ between two adjacent pulses, which matched with a repetition rate of $\sim 25.5 \mathrm{MHz}$, and the length of the laser cavity $(\sim 7.8 \mathrm{~m})$. Fig. 3d shows femtosecond pulses with the duration of 556 fs after amplification. The corresponding spectral width at halfmaximum was about $8.5 \mathrm{~nm}$ as shown in the inset of Fig. 3d. The time-bandwidth product was 0.376 , which was higher than the value of 0.315 , indicating pulses were slightly chirped. In
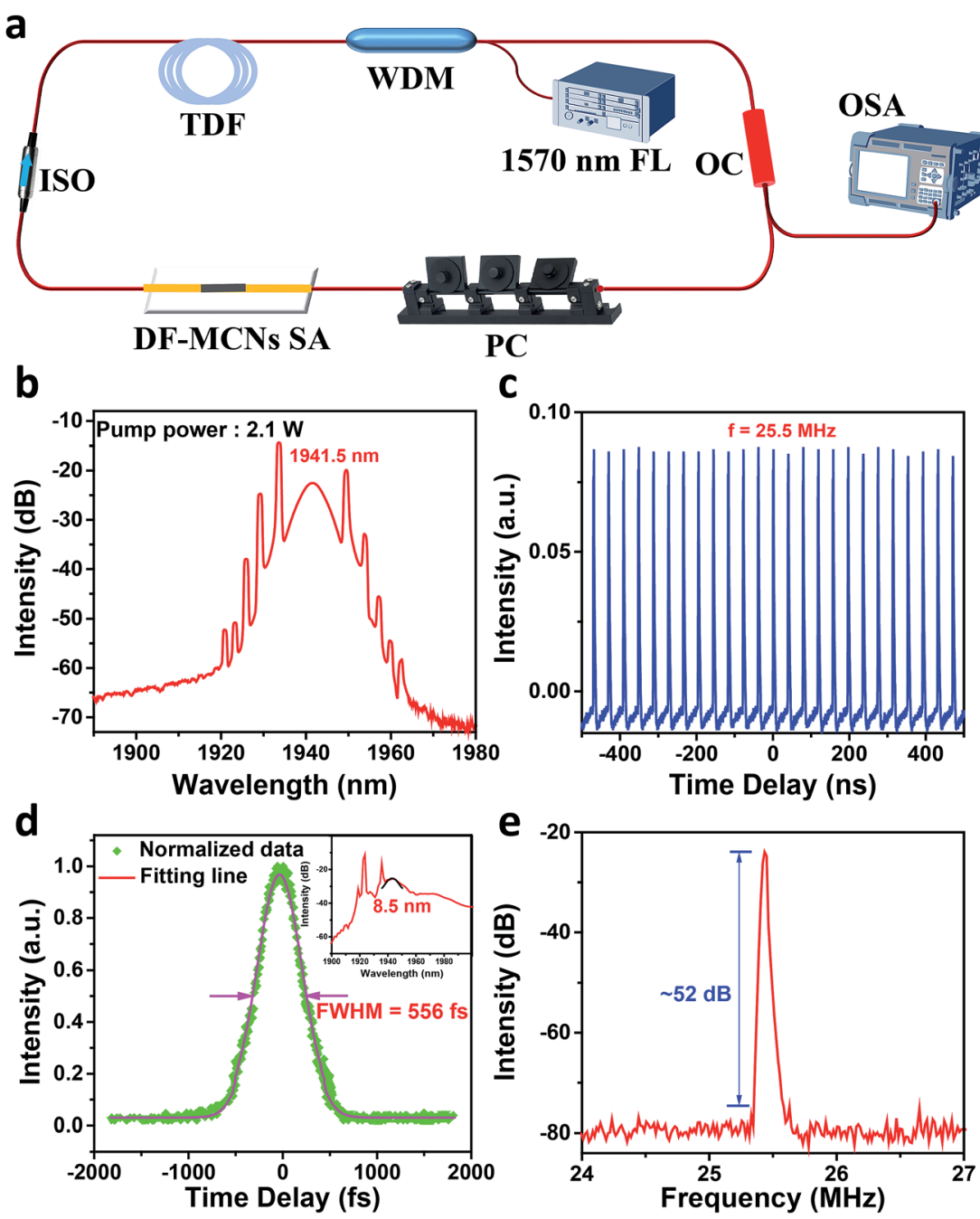

Fig. 3 (a) Experimental configuration of the TDFL. (b) The emission spectrum of the mode-locked pulse. (c) Measured pulse train of the output pulses. (d) Autocorrelation trace of a single pulse. (e) Radio frequency spectrum for signal-to-noise measurement. 
a

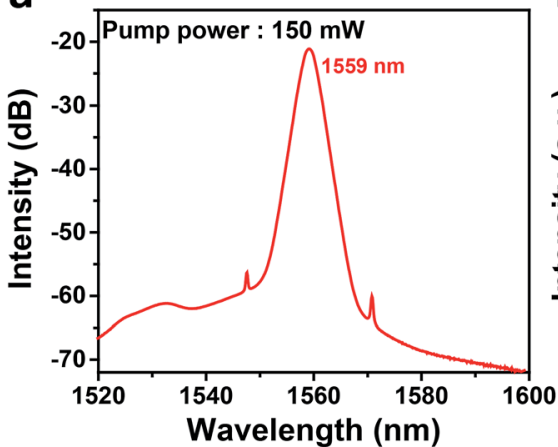

C

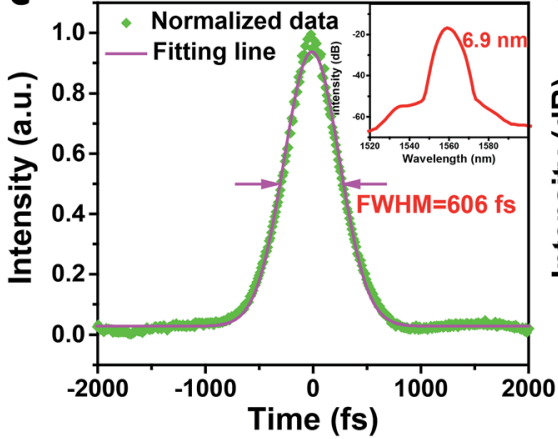

b
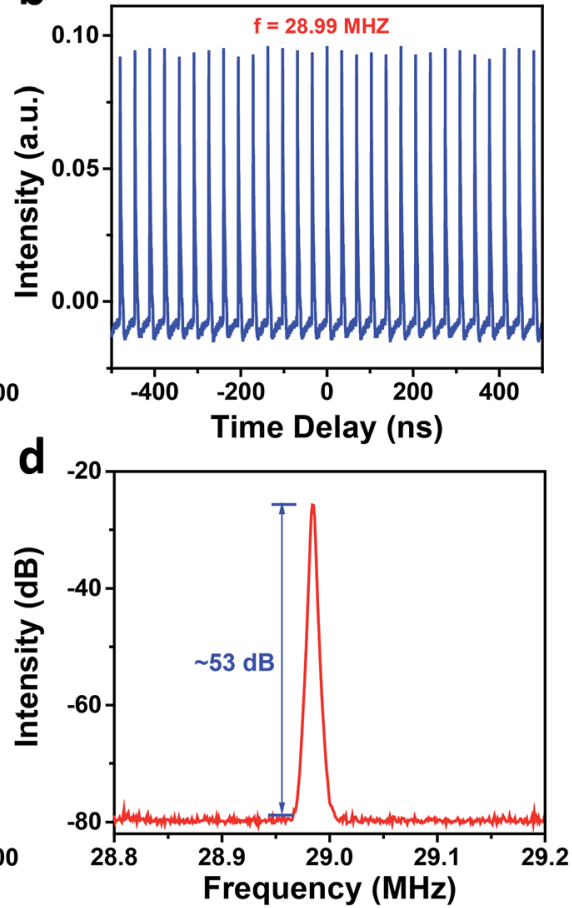

Fig. 4 (a) The emission spectrum of the mode-locked pulse. (b) Measured pulse train of the output pulses. (c) Autocorrelation trace of a single pulse. (d) Radio frequency spectrum for signal-to-noise measurement.

addition, the output power as a function of the pump power was measured and shown in the Fig. S3a. $\dagger$ As the increase of pump power from 1.3 to $2.1 \mathrm{~W}$, the output power increases linearly from 4.13 to $38.9 \mathrm{~mW}$, and the resulting conversion efficiency is about $4.15 \%$. The maximum output power $(38.9 \mathrm{~mW})$ was limited by the maximum pump power $(\sim 2.1 \mathrm{~W})$ of the $1570 \mathrm{~nm}$ fiber laser. In the future, we will further investigate the damage threshold of DF-MCNs SA in the TDFL.

Furthermore, long term stability is also a key issue for modelocked fiber lasers based on the SA. In order to confirm the long term operation stability of DF-MCNs SA, we measured the emission spectra and output power of the mode-locked fiber laser based on DF-MCNs SA every 9 hours for 72 hours in Fig. S4a and S5a. $\dagger$ It can be seen that neither central wavelength nor output power varies. The calculated root-mean-square (RMS) value of the power fluctuation at maximal output power was $0.65 \%$. These results show that the DF-MCNs SA is suitable for constructing femtosecond lasers at $2 \mu \mathrm{m}$. To further investigate the signal-to-noise ratio, we also measured the radio frequency (RF) spectrum of the mode-locked pulses. As shown in Fig. 3e, its fundamental peak locates at the cavity repetition rate $(25.5 \mathrm{MHz})$ with a signal-to-noise ratio of $52 \mathrm{~dB}$. The strong noise suppressing capability is associated with the ultrafast carrier dynamics of the SA. This further confirms that the soliton pulses have very high quality in terms of good stability.

\subsection{Femtosecond pulses generation at $1.56 \mu \mathrm{m}$}

In order to test the broadband saturable absorption property of the fabricated DF-MCNs SA, we have also inserted the SA into the EDFL cavity (Fig. S5 $\dagger$ ). Consequently, a stable femtosecond mode-locked operation is achieved at $1559 \mathrm{~nm}$ with a repetition rate of $28.99 \mathrm{MHz}$, a pulse duration of $606 \mathrm{fs}$, and a maximum output power of $2.5 \mathrm{~mW}$ (Fig. $4 \mathrm{a}-\mathrm{c}$ and $\mathrm{S} 3 \mathrm{~b} \dagger$ ). Once the pump power exceeded $150 \mathrm{~mW}$, mode-locked EDFL became unstable and DF-MCNs SA might be damaged due to the photothermal effect. The calculated time-bandwidth product was 0.515 . The spectrum with two weak Kelly bands shows a typical feature of soliton mode-locking lasers. We also measured the emission spectra and output power 9 times at an interval of 9 hours (Fig. S4b and S5b广). The calculated RMS ( 0.46) and RF spectrum (Fig. 4d) further verify the mode-locked laser with a good stability and high signal-to-noise ratio.

\section{Conclusions}

In our previous work, we have verified that MCNs are promising carbon-based broadband SAs. However, MCNs-SAs were fabricated by filmy and microfiber-based methods in ref. 24 . The MCNs films could be damaged at high pump power. Microfiberbased SAs were not robust enough and transmission loss would be increased rapidly at the tapered region. All of the abovementioned restrain the further application of MCNs-SAs for the femtosecond pulses generation. In this paper, we proposed the DF-based method to form the MCNs-SAs with the evanescent field. DFs possess more robustness and lower transmission loss than the microfibers'. The DF could be reused many times without any harm. The thickness and the overlay length of the MCNs layer could be controlled flexibly to further optimize the 
property of the SAs. Furthermore, the pulse width was reduced from 1.54 ps to 556 fs at $2 \mu \mathrm{m}$. Especially, a mode-locked EDFL with a pulse duration of 606 fs was realized by using the DFMCNs SA. Hence, DF-MCNs SAs are preferable choice for mode-locked lasers with high performance.

In conclusion, we have demonstrated a novel SA based on MCNs and DFs for femtosecond pulses generation. The MCNs were prepared by a "silica-assisted" method. The SAs were fabricated by depositing the MCNs onto the DFs to introduce strong evanescent field. The DF-MCNs SAs possess excellent linear absorption and nonlinear saturable absorption properties covering from 400 to $3400 \mathrm{~nm}$. As the DF-MCNs SA is inserted into the laser cavities, femtosecond pulses are generated with the central wavelengths of 1559 , and $1941.5 \mathrm{~nm}$. In the future, we will try to obtain ultrashort pulse ( $<200 \mathrm{fs}$ ) based on DF-MCNs SA by optimizing the dispersion parameters of the laser cavity. Our results verify that MCNs based on DFs as the cost-effective and easily-prepared SAs would be of great importance for the stable and high-power femtosecond fiber lasers.

\section{Conflicts of interest}

There are no conflicts to declare.

\section{Acknowledgements}

National Natural Science Foundation of China (NSFC) (Grant No. 61527823, 61378004, 61605219, 61605058, 61827821, 11774132 and 11474132), the Opened Fund of the State Key Laboratory on Integrated Optoelectronics, and Tsinghua National Laboratory for Information Science and Technology Cross-Discipline Foundation, the Key Technology Research and Development Project of Jilin Province (No. 20180201120GX), the Major Science and Technology Tendering Project of Jilin Province (No. 20170203012GX), the Joint Foundation from Equipment Pre-Research and Ministry of Education (No. 6141A02022413), the Outstanding Young Talent Fund Project of Jilin Province (No. 20180520188JH), Youth Innovation Promotion Association, CAS, and the opened fund of State Key Laboratory of Laser Interaction with Matter (SKLLIM1612).

\section{References}

1 U. Keller, Nature, 2003, 424, 831-838.

2 F. W. Wise, A. Chong and W. H. Renninger, Laser Photonics Rev., 2008, 2, 58-73.

3 R. R. Gattass and E. Mazur, Nat. Photonics, 2008, 2, 219-225. 4 M. E. Fermann and I. Hartl, Nat. Photonics, 2013, 7, 868-874. 5 Z. C. Luo, M. Liu, H. Liu, X. W. Zheng, A. P. Luo, C. J. Zhao, H. Zhang, S. C. Wen and W. C. Xu, Opt. Lett., 2013, 38, 52125215.

6 F. Dausinger and F. Lichtner, Femtosecond Technology for Technical and Medical Applications, Springer, Berlin, 2004.

7 N. Nishizawa, Y. Chen, P. Hsiung, E. P. Ippen and J. G. Fujimoto, Opt. Lett., 2004, 29, 2846-2848.

8 T. Fujii, and T. Fukuchi, Laser Remote Sensing, Taylor \& Francis, Boca Raton, 2005.
9 I. Coddington, W. C. Swann, L. Nenadovic and N. R. Newbury, Nat. Photonics, 2009, 3, 351-356.

10 S. Iijima, Nature, 1991, 354, 56-58.

11 P. Avouris, M. Freitag and V. Perebeinos, Nat. Photonics, 2008, 2, 341-350.

12 A. M. Chernysheva, A. Rozhin, Y. Fedotov, C. Mou, R. Arif, S. M. Kobtsev, E. M. Dianov and S. K. Turitsyn, Nanophotonics, 2017, 6, 1-30.

13 L. Wen, F. Li and H. M. Cheng, Adv. Mater., 2016, 28, 43064337.

14 Q. L. Bao, H. Zhang, Y. Wang, Z. H. Ni, Y. L. Yan, Z. X. Shen, K. P. Loh and D. Y. Tang, Adv. Funct. Mater., 2009, 19, 30773083.

15 J. Boguslawski, J. Sotor, G. Sobon, R. Kozinski, K. Librant, M. Aksienionek, L. Lipinska and K. M. Abramski, Photonics Res., 2015, 3, 119-124.

16 M. Pawliszewska, T. Martynkien, A. Przewłoka and J. Sotor, Opt. Lett., 2018, 43, 38-41.

17 Y. Chen, P. F. Xu, M. Y. Wu, Q. S. Meng, H. R. Chen, Z. Shu, J. Wang, L. X. Zhang, Y. P. Li and J. L. Shi, Adv. Mater., 2014, 26, 4294-4301.

18 M. X. Liu, J. S. Qian, Y. H. Zhao, D. Z. Zhu, L. H. Gan and L. W. Chen, J. Mater. Chem. A, 2015, 3, 11517-11526.

19 H. Tian, Z. Lin, F. Xu, J. Zheng, X. Zhuang, Y. Mai and X. Feng, Small, 2016, 12, 3155-3163.

20 C. Y. Li, Y. Meng, S. S. Wang, M. Qian, J. X. Wang, W. Y. Lu and R. Q. Huang, ACS Nano, 2015, 9, 12096-12103.

21 G. Wang, Y. H. Sun, D. B. Li, H. W. Liang, R. H. Dong, X. L. Feng and K. Mullen, Angew. Chem., Int. Ed., 2015, 54, 15191-15196.

22 J. Chong, X. J. Zhu, W. Q. Huang, X. F. Wang and Y. Tian, Carbon, 2018, 126, 156-164.

23 L. B. Zhou, Y. Jing, Y. B. Liu, Z. H. Liu, D. Y. Gao, H. B. Chen, W. Y. Song, T. Wang, X. F. Fang, W. P. Qin, Z. Yuan, S. Dai, Z. A. Qiao and C. F. Wu, Theranostics, 2018, 8, 663-675.

24 F. Wang, Y. Jing, Z. Kang, L. B. Zhou, Z. R. Li, M. Y. Liu, T. Wang, C. F. Yao, H. B. Chen, W. P. Qin, Z. A. Qiao, G. S. Qin and C. F. Wu, Adv. Opt. Mater., 2018, 6, 1800606.

25 Y. W. Song, S. Yamashita, C. S. Goh and S. Y. Set, Opt. Lett., 2007, 32, 148-150.

26 H. Jeong, S. Y. Choi, F. Rotermund, Y. H. Cha, D. Y. Jeong and D. I. Yeom, Opt. Express, 2014, 22, 22667-22672.

27 M. Kowalczyk, J. Boguslawski, R. Zybala, K. Mars, A. Mikula, G. Sobon and J. Sotor, Opt. Mater. Express, 2016, 6, 22732282.

28 Z. A. Qiao, B. K. Guo, A. J. Binder, J. H. Chen, G. M. Veith and S. Dai, Nano Lett., 2013, 13, 207-212.

29 S. M. Tseng and C. L. Chen, Appl. Opt., 1992, 31, 3438-3447. 30 J. Wang, Y. Hernandez, M. Lotya, J. N. Coleman and W. J. Blau, Adv. Mater., 2009, 21, 2430-2435.

31 C. W. Rudy, A. Marandi, K. L. Vodopyanov and R. L. Byer, Opt. Lett., 2013, 38, 2865-2868.

32 P. Sprangle, A. Ting, J. Penano, R. Fischer and B. Hafizi, IEEE J. Quantum Electron., 2009, 45, 138-148.

33 M. Zhang, E. J. R. Kelleher, F. Torrisi, Z. Sun, T. Hasan, D. Popa, F. Wang, A. C. Ferrari, S. V. Popov and J. R. Taylor, Opt. Express, 2012, 20, 25077-25084. 